\title{
A new approach to detecting and designing living structure of urban environments
}

\author{
Bin Jiang ${ }^{1^{*}}$ (D) and Ju-Tzu Huang ${ }^{2}$
}

\begin{abstract}
Sustainable urban design or planning is not a LEGO-like assembly of prefabricated elements, but an embryo-like growth with persistent differentiation and adaptation towards a coherent whole. The coherent whole has a striking character - called living structure - that consists of far more small substructures than large ones. To detect the living structure, this paper develops a new approach for uncovering the underlying living structure of urban environments. The approach takes an urban environment as a whole and recursively decomposes it into meaningful subwholes at different levels of hierarchy (or scale) ranging from the largest to the smallest. This approach helps us not only better understand an urban environment as a living structure, but also better plan or transform the urban environment to be living or more living, or equivalently to be beautiful or more beautiful.
\end{abstract}

Keywords: Urban design or planning, Structural beauty, Space syntax, Natural streets, Life, Wholeness

In such a world, scientists would do better, the profound questions of health, wholeness, nature, ecology, and human joy, would be part of a single world view, in which it would be recognized as part of science - scientia - that is to say, knowledge - and in which scientists and artists together, speaking a common language, would take part in this joy, to the benefit of all humankind.

Christopher Alexander (2003)

\section{Introduction}

Sustainable urban design or planning is not an assembly of prefabricated elements, like LEGO, but an embryolike growth with persistent differentiation and adaptation towards a coherent whole (Alexander, 1987, 2002-2005). The coherent whole is also called living structure or

\footnotetext{
* Correspondence: bin.jiang@hig.se

${ }^{1}$ Faculty of Engineering and Sustainable Development, Division of GIScience, University of Gävle, SE-801 76 Gävle, Sweden

Full list of author information is available at the end of the article
}

wholeness, which is a physical phenomenon and mathematical structure that consists of numerous substructures with an inherent hierarchy (Alexander, 2002-2005; Jiang, 2019). Across different levels of the hierarchy, there are far more small substructures than large ones (or scaling law) (Jiang, 2015a), whereas on each level of the hierarchy, there are more or less similar substructures, so called Tobler's law. It is commonly referred to as the first law of geography, which charmingly states that everything is related to everything else, but near things are more related than distant things (Tobler, 1970). Living structure exists pervasively in our surrounding. For example, a country is a living structure because it consists of far more small cities - or substructures in general - than large ones across all scales, while cities on each scale tend to be more or less similar. A city is a living structure because it consists of far more short streets - or substructures in general - than long ones across all scales, while streets on each scale tend to be more or less similar.

The present paper develops a new approach for efficiently detecting the underlying living structure and its 
recursively defined substructures, and thereafter for effectively designing livable urban environments. The approach takes an urban environment as a whole and decomposes it into numerous subwholes in a step-bystep fashion. The whole can be said to be persistently in a recursive manner - differentiated to numerous subwholes (so called differentiation principle) with far more smalls than larges across different scales, whereas the resulting subwholes are well adapted to each other (so-called adaptation principle) with more or less similar on each of the scales. This approach is developed under the third view of space, or an organismic cosmology, first conceived by the British philosopher Whitehead (1929) and further developed by Alexander (2002-2005) that space is neither lifeless nor neutral, but a living structure capable of being more living or less living. Under this organismic world view, the world is a coherent whole rather than fragmented pieces, as currently conceived under the Cartesian world picture (Descartes 1637, 1954). This holistic view of space implies that any design or planning action at any level of scale would affect virtually the livingness or beauty of the entire Earth's surface and even beyond towards the universe.

This paper is further motivated by the state of the art of science, or urban science in particular, which is largely concerned about understanding complexity rather than creating various complex or living structures. This state of science applies not only to conventional science conceived as separated from art and humanities (Snow, 1959), but also to complexity science (Alexander, 2003; Simon, 1962) such as fractal geometry (Mandelbrot, 1983), complex networks (Newman et al., 2006), and generative science (Epstein, 1999; Wolfram, 2003), all related to complex or beautiful patterns or structures. However, none of these sciences really intend to address the issue of creating structures. In some cases, they do involve creating structures, but they are unable to address why a structure is beautiful, and how beauty the structure is. Instead, the issue of creating structures or things is commonly left to engineering or art and ascribed to a matter of opinions or personal preferences. For example, fractal geometry is able to generate fractal patterns, but when comes to how good or how beautiful a fractal pattern is, it has no formal answer. It is the same for cellular automata patterns that emerged from a set of transitional rules (Wolfram, 2003). The notion of living structure directly confronts the issue of beauty or livingness and aims not only to better understand complexity, but also to effectively create beautiful or living structures (Alexander, 2003; Gabriel \& Quillien, 2019; Jiang, 2015b; Mehaffy, 2017; Salingaros, 2012, 2013). More importantly, the created living structure can be judged objectively in terms of its degree of beauty or livingness; the higher the degree of living structure, the more beautiful the structure is (Jiang, 2019). Therefore, living structure is to beauty what temperature is to warmness.

The remainder of this paper is organized as follows. Section 2 introduces the concept of living structure from a dynamic or design point of view and shows how a painting as a living structure is transformed or differentiated in a step-by-step fashion. Section 3 presents the new approach to detecting living structure and its substructures at different levels of hierarchy (or scale) by a working example of leaf vein. Section 4 report four case studies to demonstrate its usefulness for understanding and designing living structure of urban environments. Section 5 further discusses the new approach and its implications on sustainable urban design. Finally, Section 6 concludes this paper and points to future work.

\section{Living structure and the degree of livingness or beauty}

The most profound design thought advocated by Alexander (1987, 2002-2005) and his associates such as Salingaros (2005) and Mehaffy (2017) is that a city is a coherent whole that emerged and evolved in a piecemeal fashion rather than a LEGO-like assembly of prefabricated elements. In other words, a city grows out of wholeness, another name of living structure, in a stepby-step style, very much like an embryo that grows to be a human being. This is the reason why most beautiful towns and cities of the past - such as Venice and Amsterdam - are able to convey a feeling of unity or wholeness or being organic.

Given two living structures, it is possible to objectively judge which one holds a higher degree of livingness or beauty. To make this idea clear and to further illustrate scaling law and Tobler's law, let us examine two paintings (Fig. 1) in terms of their degrees of livingness or beauty. The first painting is called Composition, by the Dutch painter Piet Mondrian (1872-1944), one of the greatest artists of the twentieth century, and famous for his paintings using simple geometric shapes and the primary colors of red, yellow, and blue. The second painting is modified from Composition and it is named Configuration for the sake of convenience. These two paintings look very similar to each other, with a slight difference in the left strip. Configuration is objectively more living or more beautiful than Composition, as illustrated in Fig. 1. Under the assembly view, both paintings are composed of seven and nine pieces or substructures. However, under the living structure view, there are respectively $18(7+$ $6+4+1)$ substructures for Composition, and $20(9+6+4+$ 1) substructures for Configuration. It is important to note that the large scales are embedded in the small scales for the living structure view. Seen from the living structure point of view, Composition is less living or less beautiful than Configuration, because the notion of far more smalls than large 


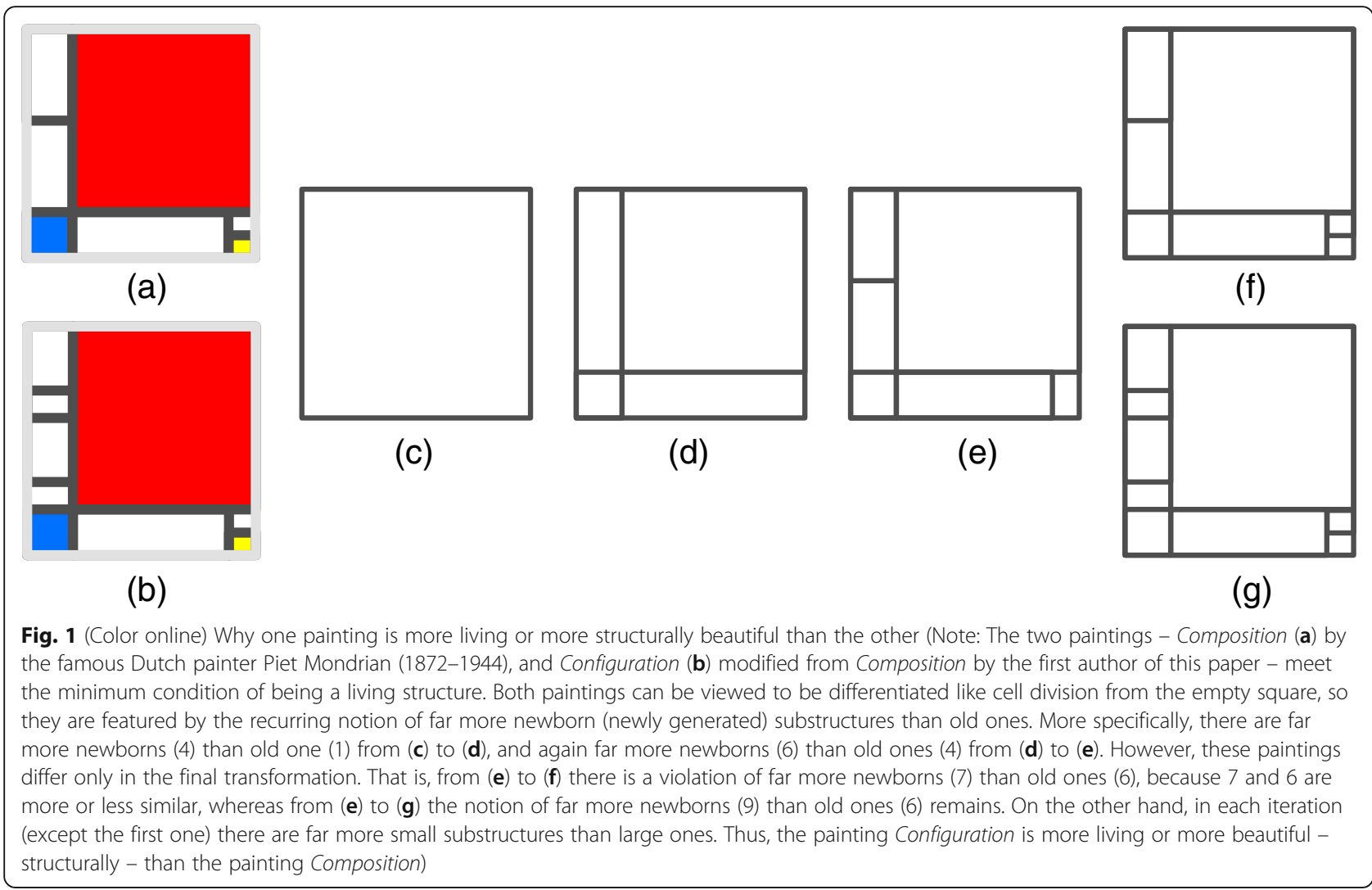

occurs only twice for the former, compared to three times for the latter. It is the difference of twice and three times that makes painting Configuration is more living or more structurally beautiful than painting Composition.

Figure 1 illustrates not only the two paintings with different degrees of beauty, but also a recursive or holistic way of seeing things as a living structure. A living structure is therefore a set of differentiated substructures at different levels of hierarchy (or scale) with far more smalls than larges. In other words, a living structure consists of far more small substructures than large ones. According to Alexander's definition of living structure, the differentiated substructures are called centers, the only building blocks of a living structure. In this paper, we replace the term center with substructure, which is simpler for better understanding living structure. That is, a living structure is the structure of the structure of the structure and so on. The recursive way of stating living structure also constitutes the foundation of the new approach to detecting living structure.

\section{A new approach to detecting living structure and its substructures}

The new approach is developed based on black and white city plans to automatically detect all of the smallest substructures, from which large substructures at upper levels of hierarchy (or scale) can be merged in a step-by-step fashion. For the lowest or smallest substructures, we use the head/tail breaks (Jiang, 2013a) to have them detected automatically. Before getting into details of the approach, let us first introduce the head/tail breaks and the degree of beauty or livingness.

\subsection{Head/tail breaks and the degree of beauty}

Head/tail breaks is an iterative function, which partitions a data as a whole around the average into the head for those big values and the tail for those small values, and then derives iteratively the head of the head of the head, and so on. To illustrate how head/tail breaks works, let us use the 10 number $[1,1 / 2,1 / 3, \ldots, 1 / 10]$ to show how it can be classified (Fig. 2). The average of the 10 number is approximately 0.29 , which partitions them into two groups: the head for those greater than the average $[1,1 / 2,1 / 3]$ and the tail for those less than the average $[1 / 4,1 / 5, \ldots, 1 / 10]$. For the three numbers in the head, the average is 0.61 , which further partitions the head into the head [1] and the tail $[1 / 2,1 / 3]$. This dataset can thus be perceived as the head [1] of the head $[1,1 / 2,1 /$ $3]$ of the dataset $[1,1 / 2,1 / 3, \ldots, 1 / 10]$.

These 10 numbers also present a very good example for illustrating the notion of living structure. First of all, the 10 numbers follow the scaling law, for the notion of far more smalls than larges recurs twice, so the ht-index is 3 (Jiang \& Yin, 2014), leading to the three classes. On 


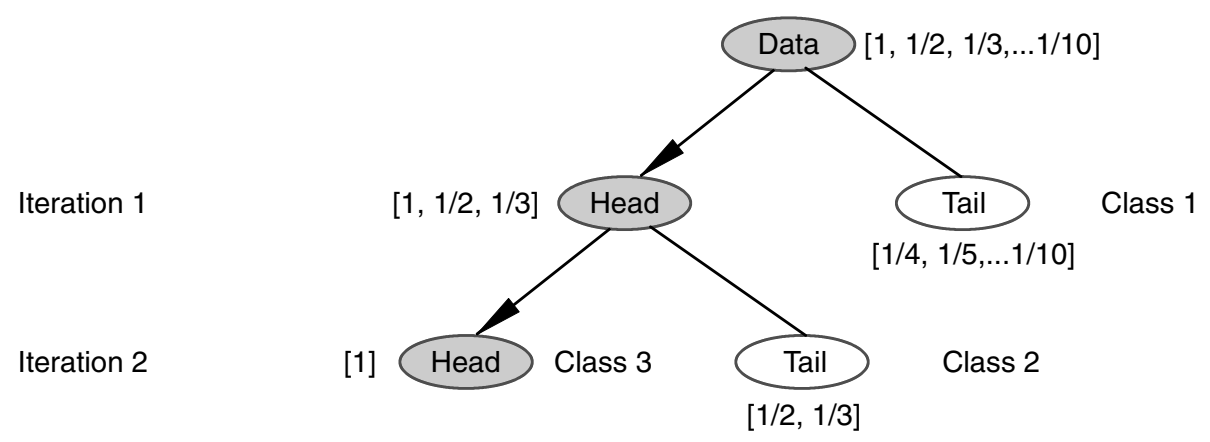

Fig. 2 Illustration of head/tail breaks classification with a simple example of the 10 numbers. (Note: The 10 numbers $[1,1 / 2,1 / 3, \ldots, 1 / 10]$ are classified into three classes: $[1 / 4,1 / 5, \ldots, 1 / 10],[1 / 2,1 / 3]$, and [1]. On the other hand, the dataset can be recursively or hierarchically perceived as the head [1] of the head $[1,1 / 2,1 / 3]$ of the data itself)

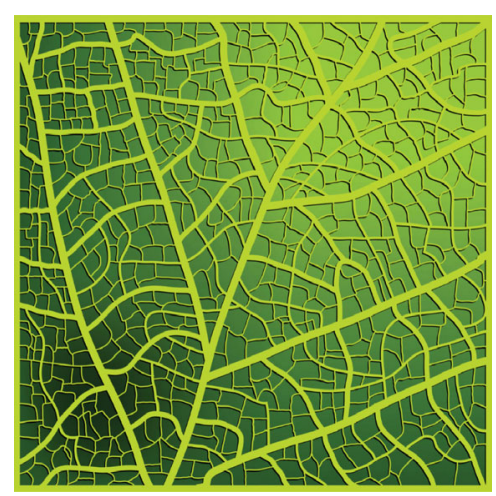

(a)

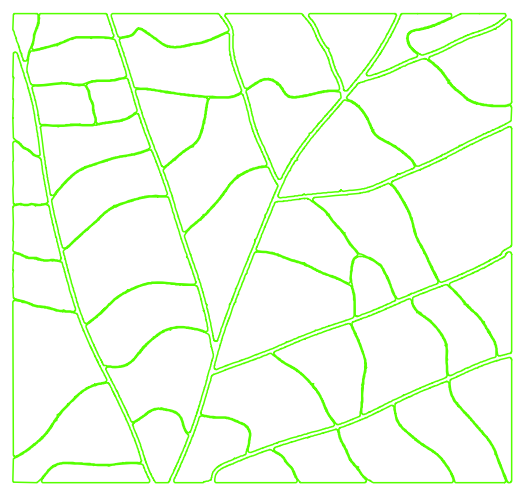

(d)

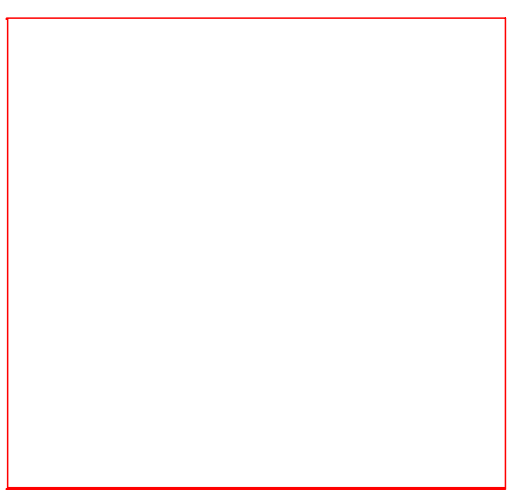

(b)

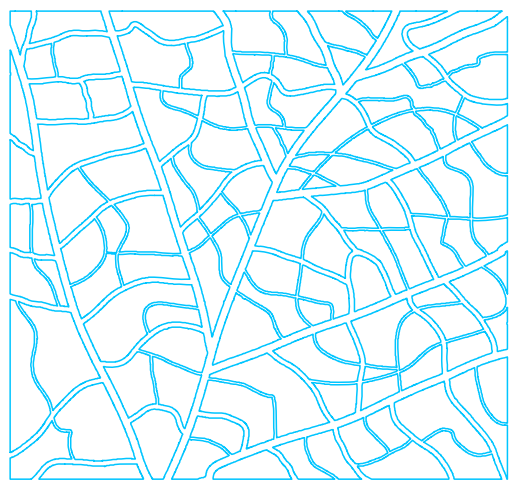

(e)

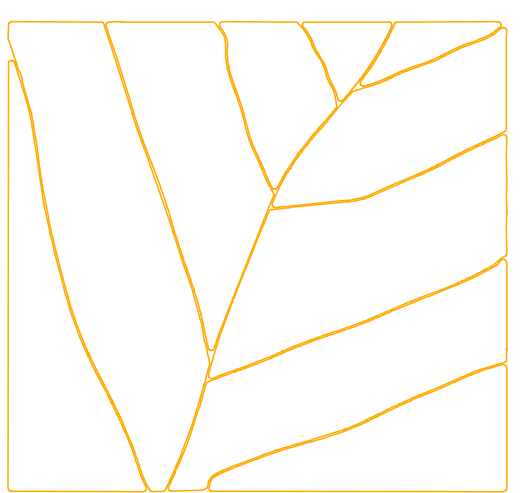

(c)

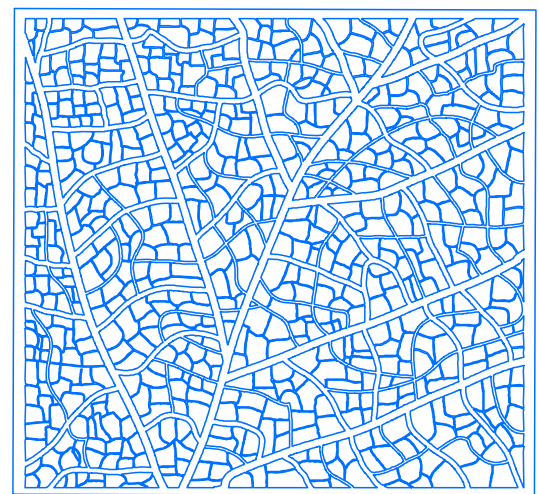

(f)

Fig. 3 (Color online) A leaf vein (a) is decomposed into many substructures (b-f). (Note: The leaf structure has five levels of scale (or classes) and is governed by two fundamental laws: the scaling law or far more small substructures than larges across all scales from the largest (Panel $\mathbf{b}$ in red) to the smallest (Panel $\mathbf{f}$ in blue); and Tobler's law or more or less similar sized substructures on each scale (one of these Panels $\mathbf{b}-\mathbf{f}$ ). For example, there are far more smalls (10) than large one (1) from Panel $\mathbf{b}$ to Panel $\mathbf{c}$, and there are far more smalls (48) than larges (10) from Panel c to Panel $\mathbf{d}$. The leaf structure can also be considered to be differentiated from a square space with adaption on each scale, thus the two principles of differentiation and adaptation. A fundamental difference between $(\mathbf{a})$ and $(\mathbf{f})$ is that the former has all five levels of scale together, while the latter only has the smallest scale. It should be noted that one could add another level of scale between (b) and (c), that is, the empty square (b) is first partitioned into two substructures (the left and the right), and then into ten substructures (c)) 
the other hand, numbers in each of the three classes are more or less similar, so they follow Tobler's law. The 10 numbers follow Zipf's law (1949) exactly rather than statistically; that is, the first largest city is twice as big as the second largest, three times as big as the third largest, and so on. The essence of Zipf's law is its statistical rather than exact nature. Strictly speaking, the 10 numbers do not follow Zipf's law, whereas its variants $\left[1+\mathrm{e}_{1}, 1 /\right.$ $2+e_{2}, 1 / 3+e_{3}, \ldots, 1 / 10+e_{10}$ ] (where $e_{1}, e_{2}, e_{3}, \ldots e_{10}$ are very small values) do follow Zipf's law. It is in this sense that the varied 10 numbers are more living or more beautiful - structurally - than the initial 10 numbers.

\subsection{A working example based on a leaf vein}

We use a leaf vein as a working example to introduce the new approach to detecting living structure and its substructures. This working example extends the above example of the 10 numbers to one million numbers (or pixels). Assuming that the leaf vein is with a color image of one million pixels (Fig. 3a), we first convert the color image to a gray-scale image (Fig. 4a) according to the formula: $\quad$ Gray $=0.299 \times$ Red $+0.587 \times$ Green $+0.114 \times$ Blue, which is commonly used the image processing literature (Poynton, 2003). Thus, there are one million pixels with gray scales between 0 and 255 . Note that these one million pixels do not follow a heavy-tailed distribution, indicating that the pixel perspective is unable to reveal the living structure. Regardless of this, we still can apply the head/tail breaks to the gray image to get multiple averages recursively (Table 1 ), from which we chose a meaningful value (171) somewhere around the second mean for delineating the smallest substructures (Fig. 3f). From these smallest substructures, large substructures at different levels of scale (or hierarchy) can be created by merging the small scales in a step-by-step fashion (Fig. 3b-e). The outcomes shown in Fig. 3 reflect very well what we human beings perceive about the inherent hierarchy of the leaf vein. The spectral coloring is adopted to show the five levels of hierarchy (or scale): red for the largest scale, blue for the smallest scale, and the other colors for the scales between the largest and the smallest. The same coloring will be used in other related figures of the paper.

The leaf vein and its inherent hierarchy effectively reflect the two laws of living structure: the scaling law and Tobler's law. First, there is the recurring notion of far more smalls than larges across all five scales (Panels b-f of Fig. 3), i.e., recurring four times so the ht-index is five, leading the five classes. Second, on each of the given scales, the substructures are more or less similar in size. From a design point of view, the leaf vein is differentiated from the empty square into numerous substructures, which are well adapted to each other. The leaf vein is living or structurally beautiful for it meets the two laws. This paper is about sustainable urban design, so we must make it very clear about the structural nature of beauty and the two laws of beauty.

The kind of beauty arises out of the living structure with far more small substructures than large ones. Let us compare the leaf vein with the Sierpinski carpet (Sierpínski, 1915) as shown in Fig. 4. Both have almost the same number of hierarchical levels, but the Sierpinski

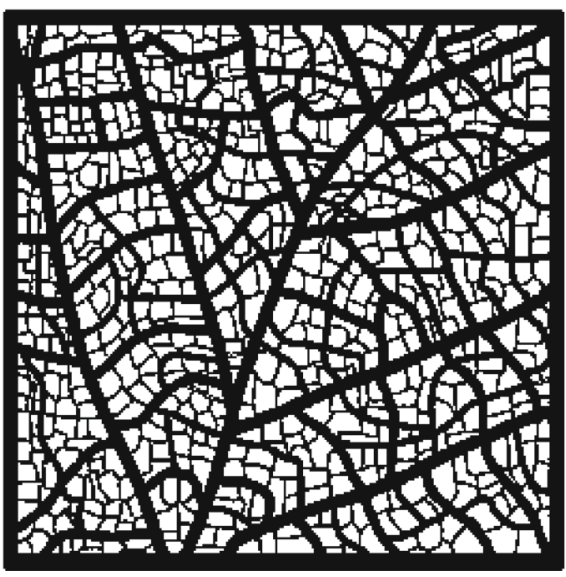

(a)

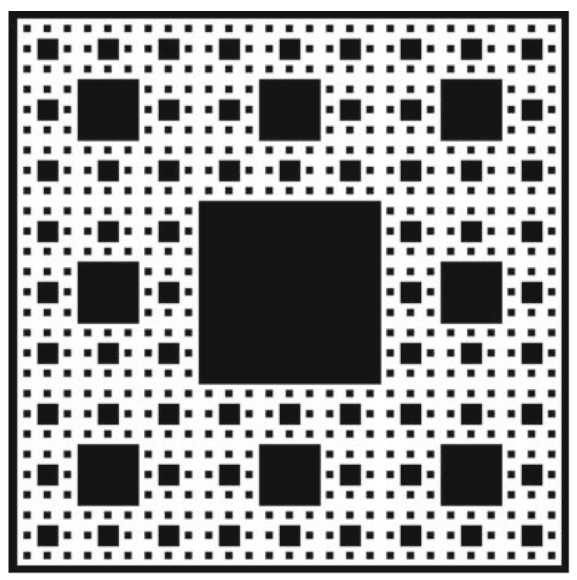

(b)

Fig. 4 The leaf vein is structurally more living or more beautiful than the Sierpinski carpet. (Note: The leaf vein (a) and the Sierpinski carpet (b) meet the scaling law with the same recurring notion of far more smalls than larges, so both are living or beautiful structures. However, the leaf vein has a higher degree of livingness or beauty than the Sierpinski carpet, because the squares on each scale are precisely the same rather than more or less similar, thus violating Tobler's law to some extent. Another obvious reason that the Sierpinski carpet is less beautiful is that its negative space is not well shaped, by which we meant a well-shaped space should be convex rather than concave. In contrast, both positive and negative spaces of the leaf vein are well shaped) 
Table 1 Head/tail breaks statistics for the gray image or the one million pixels

\begin{tabular}{llllll}
\hline \#Pixels & \#head & \%head & \#tail & \%tail & Mean \\
\hline $1,000,000$ & 523,895 & $52 \%$ & 476,105 & $48 \%$ & 125 \\
523,895 & 344,885 & $66 \%$ & 179,010 & $34 \%$ & 169 \\
344,885 & 248,693 & $72 \%$ & 96,192 & $28 \%$ & 181
\end{tabular}

The one million pixels do not follow a heavy-tailed distribution, which indicates that the pixel perspective is unable to reveal the living structure. However, the head/tail breaks still can be used to obtain the meaningful cutoff around the second mean to derive the smallest substructures

carpet is less living or less beautiful than the leaf vein. There are two reasons why the Sierpinski carpet is less living: (1) the ratio of square sizes across scales $(1 / 3,1 / 9$, $1 / 27$, and $1 / 81$ ) is exactly one third, so too strict in terms of the scaling law, and (2) the square size on each scale is exactly the same rather than more or less similar in terms of Tobler's law. It is the same issue as we remarked above on Zipf's law; the 10 numbers [1, 1/2, 1/ $3, \ldots, 1 / 10]$ are too strict to follow Zipf's law. Like Zipf's law, both the scaling law and Tobler's law should be correctly understood as statistical regularity. Strictly speaking, the Sierpinski carpet violates Tobler's law, for the substructures at each scale of the carpet are precisely the same rather than more or less similar. The Sierpinski carpet also violates the scaling law, as the notion of far more smalls than larges is too strict.

\section{Case studies}

We applied the new approach to four urban environments to further verify it and examine why it can help detect and design living structure of urban environments. The four urban environments include the village of Gassin and the town of Apt, both in south France, and two neighborhoods or districts in London: The city of London and Stockwell Station. These four cases have been well studied in the literature of space syntax (e.g.,
Hillier, 1989; Hillier et al., 1993). It was also demonstrated that the beady-ring structure of numerous convex spaces of the Gassin village represents the kind of living structure (Alexander, 2002-2005). Figure 5 shows their overall plans, which all look very organic. Note that we have deliberately not shown map scales in these plans, as we concentrated on their overall configurations rather than geometric details such as sizes and directions.

We applied the new approach to detect substructures for the four urban environments and found that they are all living structures. Table 2 reports the number of substructures at different levels of scale (or hierarchy), indicating the recurring notion of far more small substructures than large ones. The hierarchal levels vary from four to six, while the number of smallest structures changes from 26 to 462 . It is not surprising that the two neighborhoods in London are more living or more beautiful than the two in France, for they have more substructures and more hierarchical levels for these substructures. Note that we are talking in structural terms, based on the underlying plans only, without considering the vertical dimension of these urban environments.

The overall shape of the city of London looks like fish, but the organic or living nature of the environment lie in its substructures, with far more smalls than larges. For these substructures, the notion of far more smalls than larges occurs or recurs five times, so the ht-index is six, leading to the six classes or hierarchical levels. In other words, the City of London plan is a living structure consisting of far more small substructures than large ones. Figure 6 shows the six levels of scale (or hierarchy) of substructures, ranging from the largest (red) to the smallest (blue), represented by the spectral coloring suggested earlier. The inherent hierarchy of living structure can be expressed in another way: the numerous

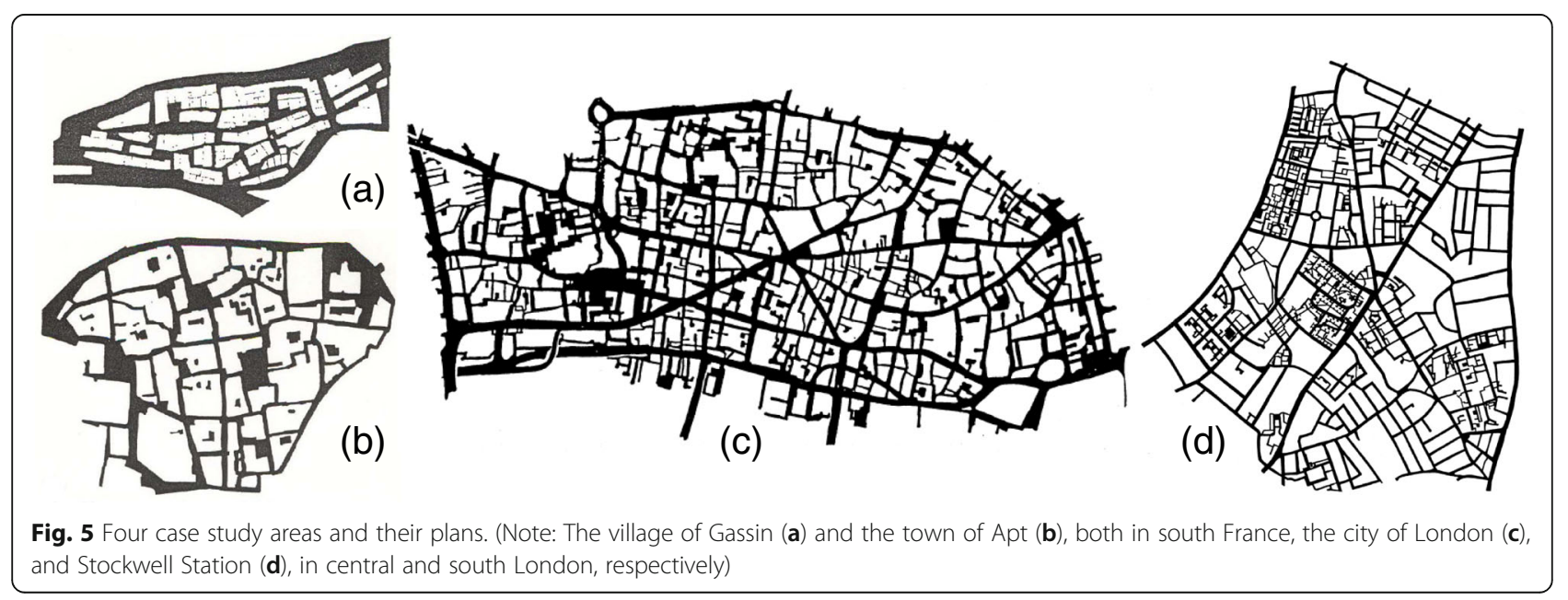


Table 2 The number of substructures (SS) at different levels of scale

\begin{tabular}{llllllll}
\hline Place & \#SS & \#SS & \#SS & \#SS & \#SS & \#SS & Ht-index \\
\hline Gassin Village & 26 & 15 & 7 & 3 & 1 & & 5 \\
Apt Town & 42 & 9 & 3 & 1 & & & 4 \\
City of London & 383 & 33 & 10 & 5 & 2 & 1 & 6 \\
Stockwell Station & 462 & 28 & 9 & 4 & 2 & 1 & 6 \\
\hline
\end{tabular}

smallest (blue), the one largest (red), and some in between the smallest and the largest (the other colors between red and blue). The recurring notion of far more smalls than larges can also be reflected in the power law distribution (Fig. 7) between the size of and the number of substructures.

The detected substructures at the different levels of hierarchy (or scale) make a better sense from a design point of view. The substructures are differentiated from scratch, an empty space in Fig. 6a. The empty space is differentiated or transformed by persistently increasing its degree of unity or wholeness in a step-by-step fashion. In other words, the empty space is filled with detailed or small structures, which makes it more and more living, or more and more beautiful. Every level of subsequent structures (or panel) is more living than the previous level (or panel) in Fig. 6, and the structure in Panel (f) is the most beautiful among the others. The city of London seems well developed towards a very beautiful structure, while part of the Stockwell Station can be developed further. This is the piecemeal design thought that has been advocated by Alexander (1987, 2002-2005) and Salingaros (2005), among many others. Now, with the new approach, we can not only diagnose an urban environment in terms of its degree of livingness or beauty, but also effectively re-design it towards more living or more beautiful.

It is important to stress the recursive nature of living structure. For example, the smallest substructures (as a whole) shown in Fig. 6f, or those shown in Fig. 3f, looks exactly as the original pattern (Fig. 5 c or Fig. 3a). However, we must realize that both Fig. $5 \mathrm{c}$ and Fig. 3a contains different levels of hierarchy (or scale). It is this living structure or recursive perspective that makes Fig. $5 \mathrm{c}$ different from Fig. 6f, and Fig. 3a different from Fig. $3 \mathrm{f}$. It is also this living structure or recursive perspective that makes the growth design thought different from the LEGO-like assembly thought.

When applying the new approach for sustainable urban design or planning, substantial consideration should be given to a larger area instead of its own area alone, which is part of the larger area. Based on the notion of wholeness or living structure, goodness of a space relies not only on its own substructures or subspaces, but also on a larger space that contains the space. The larger space should be governed by the two fundamental laws as well, implying (1) far more small substructures than large ones across scales, and (2) more or less similar substructures on each scale. A simple solution would

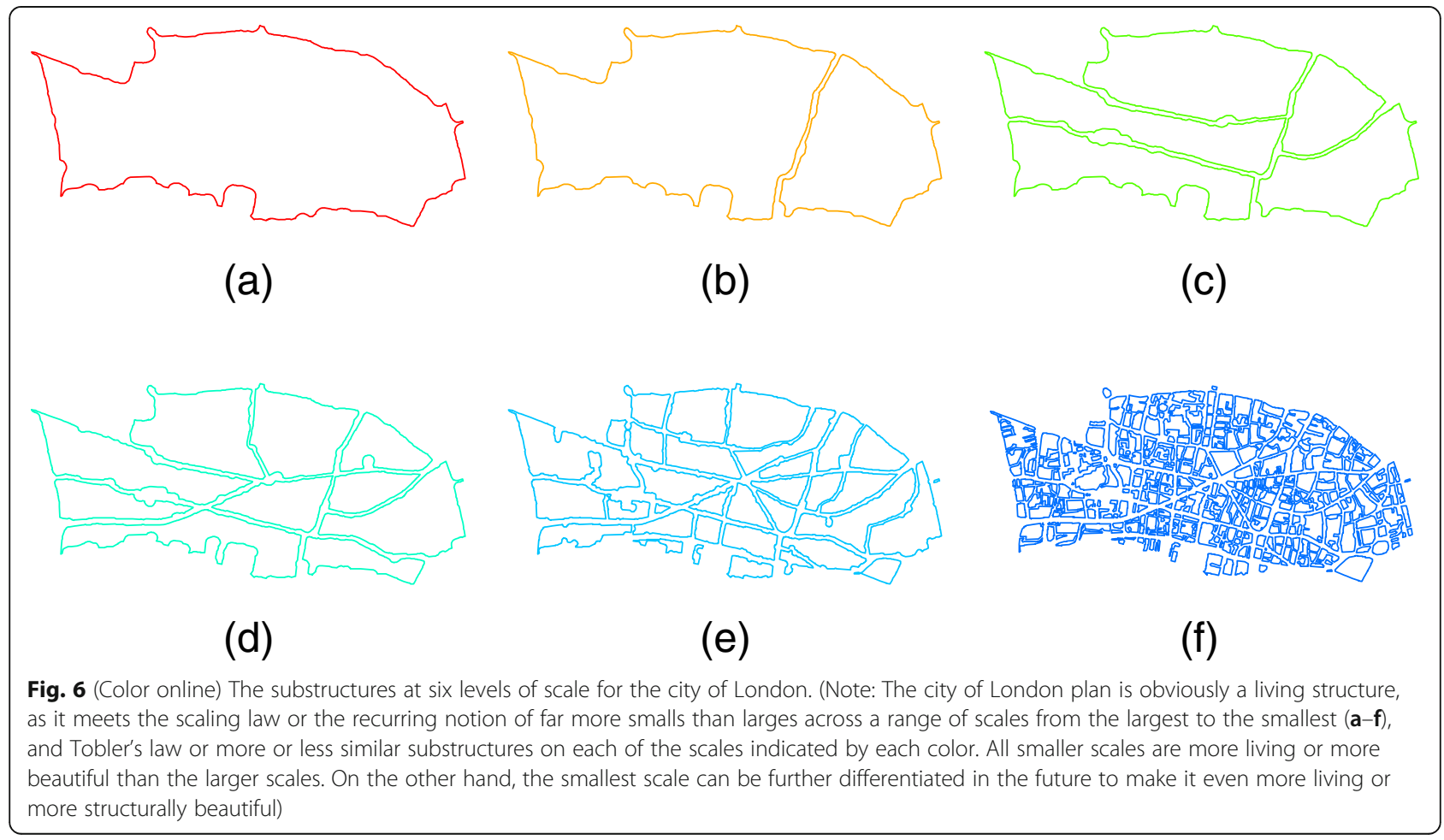




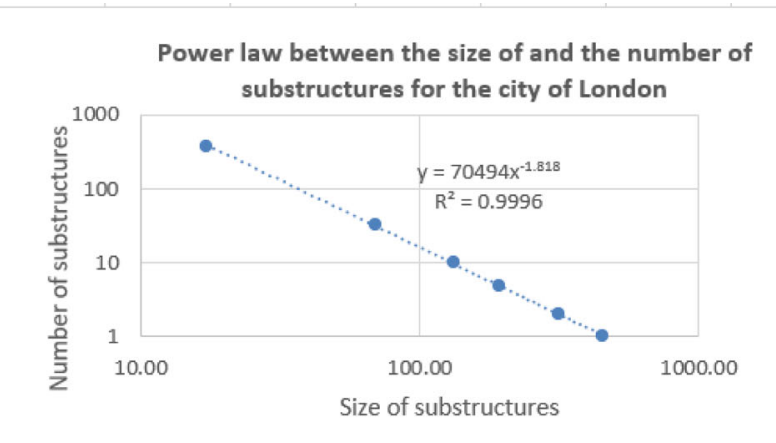

(a)
Power law between the size of and the number of substructures for the Stockwell station

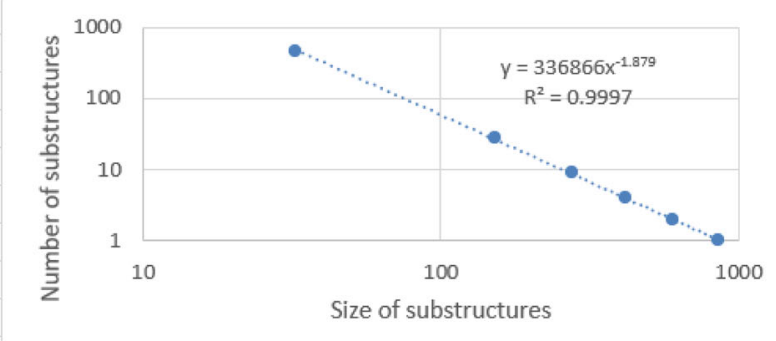

(b)

Fig. 7 (Color online) Power laws between the size and number of substructures

be to apply the approach to a substantially larger area, of which the to-be-designed area is a substructure of the larger living structure.

\section{Implications of the new approach on sustainable urban design or planning}

The new approach to detecting living structure begins with a space and decomposes it, according to its inherent hierarchy, into many substructures at different levels of hierarchy (or scale) with far more smalls than larges. These decomposed substructures enable us to see not only why an urban environment is living, but also how much living it is: the more substructures, the more living, the more hierarchal levels, the more living or more beautiful. We have already seen how the new approach can help not only achieve a better understanding of living structure of an urban environment, but also make the urban environment more living or more beautiful. In this section, we will further discuss the new approach and its implications on sustainable urban design.

While many urban theories have been developed in the past for understanding how cities structure and function, none has really aimed for urban design or planning. Even worse, urban theories have been accused of being pseudoscientific (e.g., Cuthbert, 2007; Marshall, 2012) or based on "a foundation of nonsense" (Jacobs, 1961). The new approach or the theory of living structure in general provides a larger theory that can help justify previous urban theories. For example, it is the underlying living structure that constitutes the image of the city (Jiang, 2013b; Lynch, 1960). The detected substructures at different levels of hierarchy (or scale) constitute organized complexity, as advocated by Jacobs (1961), and fundamentally resemble the model of central place theory (Christaller, 1933, 1961) in a statistical rather than strict manner (Jiang, 2018). It is the theory of living structure that was developed initially for effectively designing or planning cities (Alexander, 2002-2005).
Both geography and urban science should go beyond the understanding of complexity, towards making or remaking living or more living structures. This is the new kind of science that Alexander (2003, 2002-2005) has advocated, where both scientists and artists share a common language about the goodness of things.

We encourage the readers to conduct a thought experiment by extending the living structures studied in this paper. The city of London is a substructure of the Greater London, which is a substructure of the Great Britain, which is a substructure of the European continent, which is a substructure of the Earth's surface, and so on toward the entire universe of the largest scale. The same thought experiment can be carried out in the opposite direction; for example, the leaf vein is the superstructure of the superstructure of the superstructure and so on towards the smallest of the Planck length. In general terms, any living structure is the structure of the structure of the structure and so on. Through the thought experiment, we can achieve a better understanding of the new organismic cosmology (Whitehead, 1929), which is shared by many other scientists and philosophers. For example, the eminent quantum physicist and philosopher Bohm (1980) conceived and developed a quantum theory of wholeness, which is likened in spirit to Alexander's wholeness.

Back in 1988, Alexander and Bohm spent 2 days together exchanging their views on the conceptualization of space and the nature of the universe. There are many shared ideas and thoughts among these two great minds. Both believe that the nature of reality is more organismic than mechanistic (Whitehead, 1929) governed by the implicate order or wholeness. Both believe in the mathematical and physical structure of wholeness (Bohm, 1980), in which everything internally connects everything else, not as a local phenomenon but as a global one. Both believe in the holistic view of reality, not just on individual scales but across a range of connected 
scales from the largest to the smallest. Both believe in feelings and consciousness as something real rather than just cognitive things. All these thoughts have deep implications on sustainable urban design. Therefore, through the new developed approach, we can, should, and must adopt the growth way of urban design or planning to make our environments more living or more beautiful towards a sustainable society.

\section{Conclusion}

Built on the growth view of sustainable urban design or planning, this paper develops a new approach to detecting the underlying living structure of an urban environment. Living structure is a physical phenomenon and mathematical structure that is governed by two fundamental laws: the scaling law across all scales and Tobler's law on each of these scales. The new approach takes an urban environment as a coherent structure and decomposes it into many coherent substructures at different levels of hierarchy (or scale) with far more smalls than larges. The resulting substructures can be used to quantify or measure the degree of beauty of an urban environment: the more substructures, the more beautiful, the more hierarchical levels, the more living or more beautiful it is. Thus, the new approach offers an effective and better measure for understanding and, thereafter, for effectively designing or planning an urban environment to be living or more living. More importantly, the developed approach can help better understand the kind of problem a city is (Jacobs, 1961) and it is the problem of organized complexity.

There are three fundamental issues about a city: (1) how it looks, (2) how it works, and (3) what it ought to be. The scaling law and Tobler's law both underlie the first two issues, whereas the third issue of what it ought to be can be solved or effectively dealt with through the two design principles - differentiation and adaptation for effectively designing or planning an urban environment to be living or more living (or equivalently to be beautiful or more beautiful). Many urban design theories are largely for understanding rather than for making or remaking cities. Cities are essentially unpredictable (Alexander, 1987; Batty, 2018), but they ought to become more living or more beautiful. In this regard, the new approach provides clear guidance through the two laws and design principles. Our future work points to how the new approach can be applied to urban design or planning in collaboration with design practitioners.

\section{Acknowledgements}

This paper is abridged from the open-access paper (Jiang \& Huang, 2021). We would like to thank three anonymous referees - in particular the third one - for their insightful and deep comments. Chris de Rijke provides some help in the preparation of this paper. However, any shortcomings are ours.
Code availability

No.

\section{Authors' contributions}

Bin Jiang: Conceptualization, Data curation, Formal analysis, Funding acquisition, Investigation, Methodology, Project administration, Resources, Software, Supervision, Validation, Visualization, Writing - original draft, Writing - review \& editing. Ju-Tzu Huang: Data curation, Formal analysis, Software, Validation, Visualization, Writing - review \& editing. The author(s) read and approved the final manuscript.

\section{Funding}

The paper was partially supported by the Swedish Research Council FORMAS through the ALEXANDER project with grant number FR-2017/0009 (201700824).

\section{Availability of data and materials}

No.

\section{Declarations}

Ethics approval and consent to participate

Not applicable.

\section{Consent for publication}

Yes.

\section{Competing interests}

The author declare that they have no competing interests.

\section{Author details}

${ }^{1}$ Faculty of Engineering and Sustainable Development, Division of GIScience, University of Gävle, SE-801 76 Gävle, Sweden. ²Department of Real Estate and Built Environment, National Taipei University, 237 New Taipei, Taiwan.

Received: 19 May 2021 Accepted: 28 June 2021

Published online: 06 July 2021

\section{References}

Alexander, C. (1987). A new theory of Urban Design. Oxford University Press.

Alexander, C. (2002-2005). The nature of order: An essay on the art of building and the nature of the universe. Center for Environmental Structure.

Alexander C. (2003), New Concepts in Complexity Theory: Arising from studies in the field of architecture, an overview of the four books of The Nature of Order with emphasis on the scientific problems which are raised, http://na tureoforder.com/library/scientific-introduction.pdf

Batty, M. (2018). Inventing future cities. The MIT Press.

Bohm, D. (1980). Wholeness and the implicate order. Routledge.

Christaller W. (1933, 1966), Central Places in Southern Germany, Prentice Hall.

Cuthbert, A. R. (2007). Urban design: Requiem for an era-review and critique of the last 50 years. Urban Design International, 12(4), 177-223. https://doi.org/1 0.1057/palgrave.udi.9000200.

Descartes R. (1637, 1954), The Geometry of Rene Descartes, translated by Smith D. E., and Latham M. L, Dover Publications.

Epstein, J. M. (1999). Agent based computational models and generative social science. Complexity, 4(5), 41-60. https://doi.org/10.1002/(SICI)1099-0526(1 99905/06)4:5<41::AID-CPLX9>3.0.CO;2-F.

Gabriel, R. P., \& Quillien, J. (2019). A search for beauty/a struggle with complexity: Christopher Alexander. Urban Science, 3(2), 64. https://doi.org/10.3390/urba nsci3020064.

Hillier, B. (1989). The architecture of the urban object. Ekistics, 56(334/335), 5-21.

Hillier, B., Penn, A., Hanson, J., Grajewski, T., \& Xu, J. (1993). Natural movement: Or configuration and attraction in urban pedestrian movement. Environment and Planning B: Planning and Design, 20(1), 29-66. https://doi.org/10.1068/b2 00029

Jacobs, J. (1961). The death and life of great American cities. Random House.

Jiang, B. (2013a). Head/tail breaks: A new classification scheme for data with a heavy-tailed distribution. The Professional Geographer, 65(3), 482-494. https:// doi.org/10.1080/00330124.2012.700499. 
Jiang, B. (2013b). The image of the city out of the underlying scaling of city artifacts or locations. Annals of the Association of American Geographers, 103(6), 1552-1566. https://doi.org/10.1080/00045608.2013.779503.

Jiang, B. (2015a). Geospatial analysis requires a different way of thinking: The problem of spatial heterogeneity. GeoJournal, 80(1), 1-13. https://doi.org/10.1 007/s10708-014-9537-y.

Jiang, B. (2015b). Wholeness as a hierarchical graph to capture the nature of space. International Journal of Geographical Information Science, 29(9), 1632 1648. https://doi.org/10.1080/13658816.2015.1038542.

Jiang, B. (2018). A topological representation for taking cities as a coherent whole. Geographical Analysis, 50(3), 298-313. https://doi.org/10.1111/gea n. 12145 .

Jiang, B. (2019). Living structure down to earth and up to heaven: Christopher Alexander. Urban Science, 3(3), $96 \mathrm{https} / / \mathrm{hm} w . m d p i . c o m / 2413-8851 / 3 / 3 / 96$. Reprinted in the magazine Coordinates, March and April issues, 29-38, 12-17, 2020.

Jiang, B., \& Huang, J. (2021). A new approach to detecting and designing living structure of urban environments. Computers, Environment and Urban Systems, $88,1-10$.

Jiang, B., \& Yin, J. (2014). Ht-index for quantifying the fractal or scaling structure of geographic features. Annals of the Association of American Geographers, 104(3), 530-541. https://doi.org/10.1080/00045608.2013.834239.

Lynch, K. (1960). The image of the City. The MIT Press.

Mandelbrot, B. B. (1983). The Fractal Geometry of Nature. W. H. Freeman and Co.

Marshall, S. (2012). Science, pseudo-science and urban design. Urban Design International, 17(4), 257-271. https://doi.org/10.1057/udi.2012.22.

Mehaffy, M. (2017). Cities alive: Jane Jacobs, Christopher Alexander, and the roots of the new urban renaissance. Sustasis Press.

Newman, M., Barabási, A.-L., \& Watts, D. J. (2006). The Structure and Dynamics of Networks. Princeton University Press.

Poynton, C. (2003). Digital video and HDTV algorithms and interfaces. Morgan Kaufmann Publishers.

Salingaros, N. A. (2005). Principles of urban structure. Techne.

Salingaros, N. A. (2012). United architectural theory: Form, language, complexity. Vajra Books.

Salingaros, N. A. (2013). Algorithmic sustainable design: Twelve lectures on architecture. Sustasis Press.

Sierpínski, W. (1915). Sur une courbe dont tout point est un point de ramification Comptes Rendus Hebdomadaires des Séances de l'Académie des Sciences, 160, 302-305.

Simon, H. A. (1962). The architecture of complexity. Proceedings of the American Philosophical Society, 106(6), 467-482.

Snow, C. P. (1959). The two cultures and the scientific revolution. Cambridge University Press.

Tobler, W. (1970). A computer movie simulating urban growth in the Detroit region. Economic Geography, 46(2), 234-240. https://doi.org/10.2307/143141.

Whitehead, A. N. (1929). Process and reality: An essay in cosmology. The Free Press.

Wolfram, S. (2003). A new kind of science. Wolfram Media.

Zipf, G. K. (1949). Human behaviour and the principles of least effort. Addison Wesley.

\section{Publisher's Note}

Springer Nature remains neutral with regard to jurisdictional claims in published maps and institutional affiliations. 